\title{
A simple model for counter-rotating ring currents in $[n]$ circulenes
}

\author{
A. Acocella ${ }^{\text {a }}$, R.W.A. Havenith ${ }^{\text {a }}$, E. Steiner ${ }^{\text {a }}$, P.W. Fowler ${ }^{\text {a,*, }}$, \\ L.W. Jenneskens ${ }^{b, 1}$ \\ ${ }^{a}$ School of Chemistry, University of Exeter, Stocker Road, Exeter EX4 4QD, UK \\ ${ }^{\mathrm{b}}$ Department of Physical Organic Chemistry, Debye Institute, Utrecht University, Padualaan 8, 3584 CH Utrecht, The Netherlands
}

Received 21 June 2002

\begin{abstract}
Ab initio current density maps for [7]circulene computed using the ipsocentric CTOCD-DZ/6-31G**//RHF/6-31G** method show that in both planar [3a $\left.\left(\mathrm{D}_{7 \mathrm{~h}}\right)\right]$ and non-planar, saddle-shaped $\left[3 \mathbf{b}\left(\mathrm{C}_{2}\right)\right]$ geometries this molecule sustains similar counter-rotating rim (diatropic) and hub (strongly paratropic) currents. A survey of the orbital contributions to the current density reveals that 3 like corannulene (1) is a $4 d+4 p$ system with just eight magnetically active and 20 magnetically inactive $\pi$ electrons. The graph-theoretical Hückel-London model describes the ring-current patterns of $[n]$ circulenes in terms of a coupling strength (the ratio of radial and tangential resonance integrals) that charts the variation from the decoupled con-rotating currents of the $[n]$ annulene-within-an- $[\mathrm{m}]$ annulene model to the fully coupled counter-rotating currents of the real $[n]$ circulene. (c) 2002 Elsevier Science B.V. All rights reserved.
\end{abstract}

\section{Introduction}

Ring currents in mono- and polycyclic conjugated systems, though not directly observable, are inferred through their manifestation in NMR spectroscopy and measurements of magnetic anisotropy [1,2]. Fortunately, modern ab initio distributed-origin methods at the coupled Hartree-Fock level, such as the ipsocentric [3] CTOCD-DZ (continuous transformation of the

\footnotetext{
${ }^{*}$ Corresponding author. Fax: +44-1392-263-434.

E-mail addresses: p.w.fowler@exeter.ac.uk (P.W. Fowler), jennesk@chem.uu.nl (L.W. Jenneskens).

${ }^{1}$ Also corresponding author. Fax: +31-30-253-4533.
}

origin of current density; diamagnetic zero variant) method $[4,5]$, now allow the direct visualisation of the induced ring current density in computations of modest cost. The accuracy of the distributed-origin maps produced in this way is confirmed by calculation of integrated global and local magnetic response properties. Recent work with this method includes the mapping of induced current density in the bowl-shaped [5]circulene, corannulene $\left(\mathbf{1}, \mathrm{C}_{5 \mathrm{v}}\right)$ and the planar [6]circulene, coronene (2, $\mathrm{D}_{6 \mathrm{~h}}$ ) [6] (Fig. 1). For 1 and $\mathbf{2}$ similar counter-rotating ring-current patterns are found: each molecule sustains a diatropic current around its outer perimeter and a paratropic current around the central ring. This 'rim and hub' pattern contradicts predictions based on the frequently 

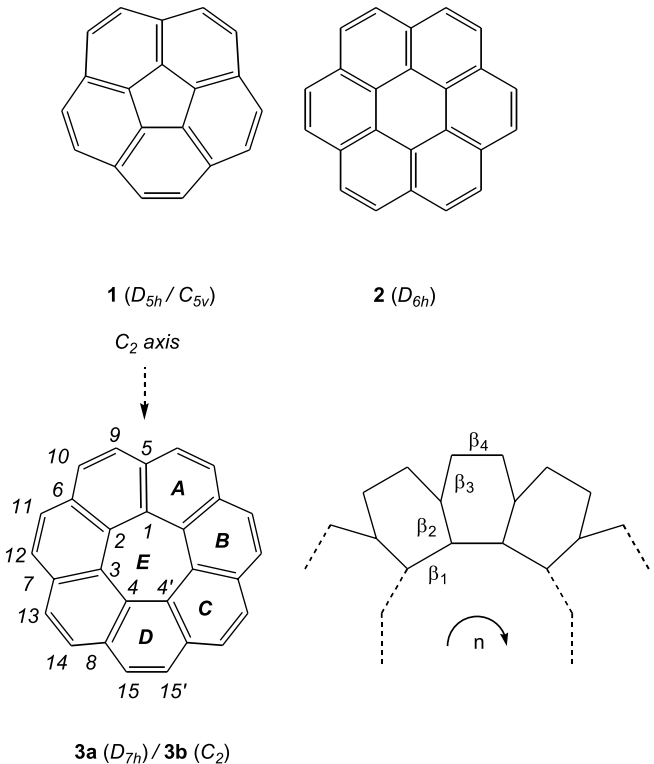

Fig. 1. Planar $\left(\mathrm{D}_{5 \mathrm{~h}}\right)$ and bowl-shaped $\left(\mathrm{C}_{5 \mathrm{v}}\right)$ corannulene (1), planar $\left(D_{6 h}\right)$ coronene $(2)$, planar $\left(D_{7 h}\right)$ and saddle-shaped $\left(C_{2}\right)$ [7]circulene (3a and 3b), and schematic $[n]$ annulene-within-an$[m]$ annulene with the four independent Hückel $\beta$ bond parameters $\beta_{1}, \beta_{2}\left(=\beta_{\text {spoke }}\right), \beta_{3}$ and $\beta_{4}$.

used ' $[n]$ annulene-within-an- $[m]$ annulene' model [7], according to which both $\mathbf{1}$ and $\mathbf{2}$ would be represented by two decoupled mono-cyclic $4 n+2$ $\pi$-electron annulenes $[\mathbf{1}, n=14(\mathrm{rim})$ and $m=6$ (hub), and 2, $n=18$ (rim) and $m=6$ (hub)], and for which con-rotating diatropic ring currents along the rim and hub are expected. As shown by the $\mathrm{ab}$ initio current density maps [6], and the analysis of orbital contributions to the total current density [3], the proposition that $[n]$ circulenes behave magnetically as decoupled concentric annulenes is no longer tenable.

In this Letter we report new current density maps and integrated magnetic properties for [7]circulene (3) [8], a compound which differs from its homologues $\mathbf{1}$ and $\mathbf{2}$ by its complex dynamics. Whilst 2 has a $\mathrm{D}_{6 \mathrm{~h}}$ structure both in solution and in the solid state [9], $\mathbf{1}$ is bowl-shaped in the solid state [10] but has a time-averaged planar $\mathrm{D}_{5 \mathrm{~h}}$ structure in solution (as established by temperature-dependent ${ }^{1} \mathrm{H}$ NMR) due to rapid bowl-to-bowl interconversion [11,12]. In contrast, 3 has a saddle-shaped structure of $\mathrm{C}_{2}$ symmetry (3b) in the solid state [8] and a time-averaged planar structure of $\mathrm{D}_{7 \mathrm{~h}}$ symmetry (3a) in solution (with one ${ }^{1} \mathrm{H}$ and three ${ }^{13} \mathrm{C}$ NMR resonances), which is not frozen out even at $-90{ }^{\circ} \mathrm{C}$ [13] (Fig. 1). Previous ab initio calculations at the RHF level using various basis sets on both $\mathbf{3 a}\left(\mathrm{D}_{7 \mathrm{~h}}\right)$ and 3b $\left(\mathrm{C}_{2}\right)$ have indicated that $\mathbf{3}$ is a floppy molecule with a notoriously shallow corrugation potential [14] (see also [15]). We show here that [7]circulene (3), despite its floppiness, sustains the same global pattern of counter-rotating currents as corannulene (1) and coronene (2), and we provide a graph-theoretical model that accounts for this behaviour of $[n]$ circulenes in general (see also [16]). The simple model gives a one-parameter continuum of descriptions from the $[n]$ annulenewithin-an- $[m]$ annulene to the strongly coupled $[n]$ circulenes.

\section{Methods}

\subsection{Structure of [7]circulene $\mathbf{3 a}-\boldsymbol{b}$}

It is known that the maximally symmetric $\mathrm{D}_{7 \mathrm{~h}}$ planar form of [7]circulene, 3a, does not occupy a local minimum on the potential energy surface. A nearby saddle-shaped structure of $\mathrm{C}_{2}$ symmetry, 3b, lies ca. $35 \mathrm{~kJ} \mathrm{~mol}^{-1}$ [14] lower in energy and compares closely with the experimental structure obtained from low-temperature X-ray measurements [8], although the precise position of the minimum is difficult to establish because of the extremely low energy of the corrugating modes [14]. The geometry of 3 was optimised at the RHF/ 6-31G** level under the constraints of $\mathrm{D}_{7 \mathrm{~h}}$ and $\mathrm{C}_{2}$ symmetry, respectively, using GAMESS-UK [17]. The geometries, energies, energy difference $\left[\Delta E_{\text {tot }}\right.$ $\left.(3 \mathbf{a}-\mathbf{3 b}) 35.6 \mathrm{~kJ} \mathrm{~mol}^{-1}\right]$ and imaginary frequencies found for 3a and 3b [3a, 60i (doubly degenerate), $62 \mathrm{i}$ and $51 \mathrm{i} \mathrm{cm} \mathrm{cm}^{-1}$, and $3 \mathrm{~b}, 7 \mathrm{i} \mathrm{cm}^{-1}$ ] are in good agreement with those obtained in the previous computational study [14] (see also Table 1). In passing we note that a change of method from RHF/6-31G** to B3LYP/6-31G** does not influence the results. Here we will compare current density maps for both planar and non-planar forms $\mathbf{3 a}$ and $\mathbf{3 b}$. 
Table 1

RHF/6-31G** carbon-carbon bond lengths (in $\AA$ ) for 3a ( $\mathrm{D}_{7 \mathrm{~h}}$ ) and $3 \mathbf{b}\left(\mathrm{C}_{2}\right)$

\begin{tabular}{|c|c|c|c|}
\hline Bond length $^{\mathrm{a}}$ & $3 \mathbf{a}$ & $3 b$ & $3 b(a v)$ \\
\hline $1-2$ & & 1.465 & \\
\hline $2-3$ & & 1.468 & \\
\hline $3-4$ & 1.485 & 1.462 & $\begin{array}{l}1.466 \\
\left(1.461^{b}\right)\end{array}$ \\
\hline $4-4^{\prime}$ & & 1.469 & \\
\hline $1-5$ & & 1.412 & \\
\hline $2-6$ & & 1.409 & \\
\hline $3-7$ & 1.423 & 1.411 & $\begin{array}{l}1.411 \\
\left(1.430^{\mathrm{b}}\right)\end{array}$ \\
\hline $4-8$ & & 1.410 & \\
\hline $5-9$ & & 1.420 & \\
\hline $6-10$ & & 1.421 & \\
\hline $6-11$ & & 1.423 & \\
\hline $7-12$ & 1.416 & 1.422 & $\begin{array}{l}1.421 \\
(1.422)^{b}\end{array}$ \\
\hline $7-13$ & & 1.419 & \\
\hline $8-14$ & & 1.419 & \\
\hline $8-15$ & & 1.423 & \\
\hline $9-10$ & & 1.334 & \\
\hline $11-12$ & & 1.333 & \\
\hline $13-14$ & 1.323 & 1.334 & $\begin{array}{l}1.334 \\
\left(1.344^{b}\right)\end{array}$ \\
\hline $15-15^{\prime}$ & & 1.334 & \\
\hline
\end{tabular}

${ }^{a}$ See Fig. 1.

${ }^{\mathrm{b}}$ Average bond lengths as reported for the low-temperature X-ray structure of $\mathbf{3 b}\left(\mathrm{C}_{2}\right)$ [8].

\subsection{Magnetic properties}

The current density maps and integrated magnetic properties were computed using distributedorigin ab initio methods, in the $6-31 \mathrm{G}^{* *}$ basis set, at the coupled Hartree-Fock level, with the Sysmo program [18]. The advantages of the CTOCD-DZ method for current density and the CTOCD-PZ2 (paramagnetic zero) variant for integrated magnetic properties have been well rehearsed in the literature $[6,16,19,20]$. For planar 3a the $\sigma-, \pi$ - and total $(\sigma+\pi)$ current densities induced by a unit magnetic field acting along the principal axis are plotted in a plane $1 a_{0}$ above that of the central ring (that is, close to the maximum $\pi$ density and where current flow is effectively parallel to the molecular plane [21]). The contours denote the modulus of the current density, and the vectors represent in-plane projections of current.
In the case of non-planar $\mathbf{3 b}$ the total current density map was plotted in a 'mean' plane (the inertial plane that contains the $\mathrm{C}_{2}$ axis and lies normal to the large-moment principal axis of the carbon framework). Orbital contributions were also mapped for the planar structure. In all plots, diamagnetic circulation is shown anti-clockwise and paramagnetic circulation clockwise. Integral properties (magnetisability, shieldings at nuclei and ring centres) were computed in the CTOCDPZ2 method [19,20].

\section{Results and discussion}

\subsection{Current density maps}

The maps for induced current density for $\mathbf{3 a}$ are presented in Fig. 2. The $\pi$-only current density map for this molecule (Fig. 2a) shows the same qualitative features as those for the smaller $[n]$ circulenes $1(n=5)$ and $2(n=6)$ [6]. A strongly diatropic circulation flows around the perimeter of the carbon framework and a paratropic circulation flows around the inner seven-membered ring. Comparison of the three cases suggests a dependence of the global pattern on $n$, the size of the central ring. In (planarised) corannulene (1) and in [7]circulene (3a), the paratropic central current is stronger than the diatropic perimeter current. When $n$ is even as in coronene (2), the reverse is true [6]. This comparison can be put on a numerical basis by scaling the maximum current vectors $|j|$ in each circulation by the maximum current calculated for benzene $\left|j_{\text {benzene }}\right|$ with the same approach, basis set and plotting plane $\left[\left|j_{\text {rim }} / j_{\text {benzene }}\right|\right.$ : 0.83 (planar 1, $n=5), 1.29(\mathbf{2}, n=6)$ and $0.90(\mathbf{3 a}$, $n=7)$ and $\left|j_{\text {hub }} / j_{\text {benzene }}\right|: 1.19$ (planar $\left.\mathbf{1}, n=5\right), 0.67$ $(\mathbf{2}, n=6)$ and $1.42(\mathbf{3 a}, n=7)$ giving $\left|j_{\text {rim }} / j_{\text {hub }}\right|: 0.70$ (planar 1, $n=5), 1.94(\mathbf{2}, n=6)$ and 0.63 (3a, $n=7)]$.

Inclusion of the $\sigma$ electrons in the total $(\sigma+\pi)$ map (Fig. 2b) enhances both the perimeter and central currents without changing their directions. In addition, extra minor features appear in the total current density, i.e., local circulations around the radial carbon-carbon bonds, which are forbidden by symmetry in a pure $\pi$ model [22-24], 

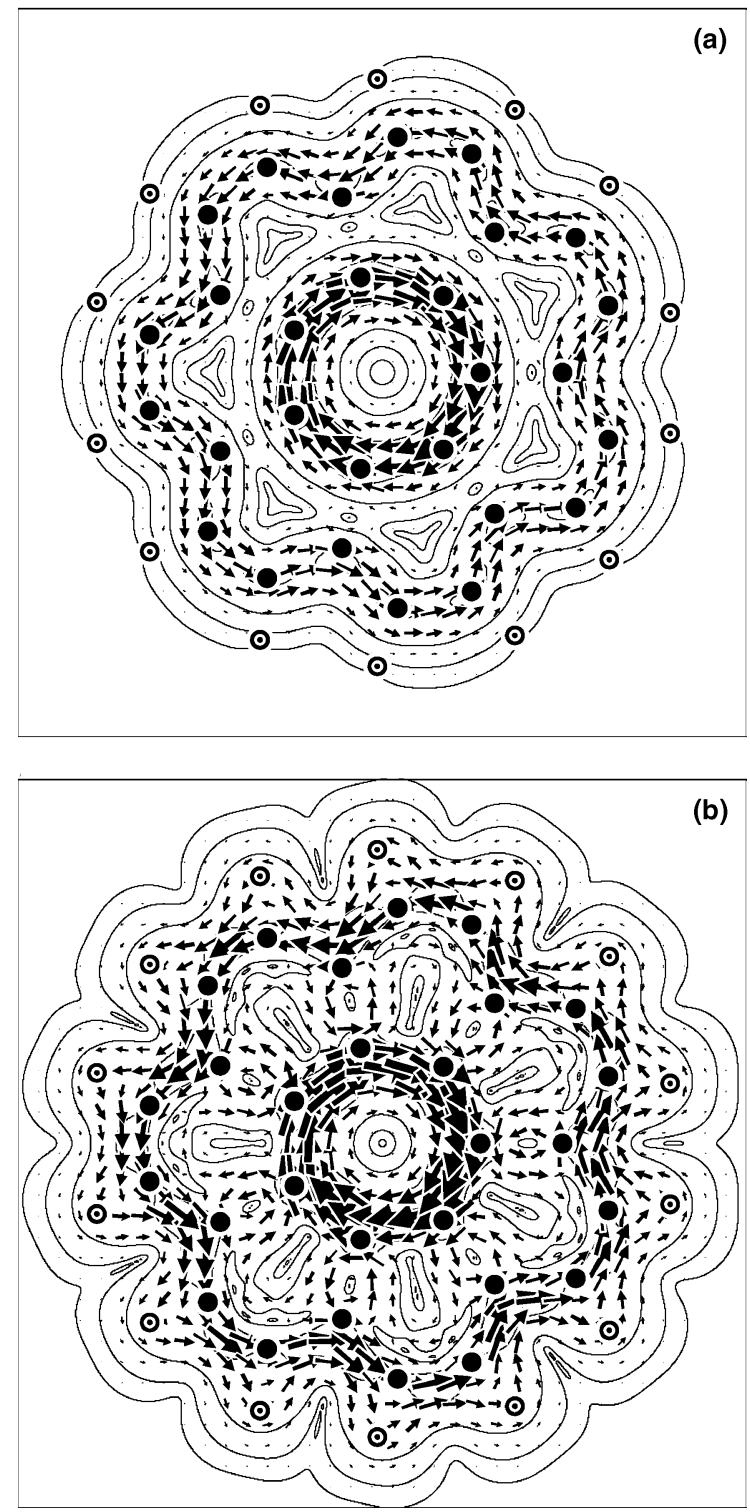

Fig. 2. Computed current density maps for planar [7]circulene 3a $\left(D_{7 \mathrm{~h}}\right)$; (a) $\pi$-only and (b) total $(\sigma+\pi)$ induced current density plotted $1 a_{0}$ above the molecular plane (carbon $\bullet$ ) and hydrogen $\odot$ ).

and paramagnetic vortices are introduced at the centres of all rings as the cumulative effect of these local $\sigma$-bond circulations.

One of the advantages of the ipsocentric method is that it allows an informative breakdown of the computed current density into orbital contribu- tions governed by symmetry and energy selection rules [3]. Diatropic currents arise from translationally allowed and paratropic currents from rotationally allowed virtual excitations of occupied orbitals. As the transition integral of any such excitation is weighted by the inverse energy difference, the frontier orbitals are typically the dominant contributors. The analysis for planar corannulene (1) and coronene (2) yields a particularly simple few-electron picture in each case [3]. In planar $\mathrm{D}_{5 \mathrm{~h}}$ corannulene (1), eight $\pi$ electrons are responsible for the $\pi$ ring currents: the $2 \mathrm{e}_{1}^{\prime \prime} \mathrm{LUMO}$ (RHF/6-31G** $0.06 \mathrm{E}_{\mathrm{h}}$ ) pair of virtual orbitals can be accessed via a rotational transition from the $2 \mathrm{e}_{1}^{\prime \prime}$ HOMO (RHF/6-31G** $-0.29 \quad \mathrm{E}_{\mathrm{h}}$ ) and via a translational transition from the near-degenerate 2e $\mathrm{e}_{2}^{\prime \prime} \mathrm{HOMO}-1$ (RHF/6-31G** $\left.-0.30 E_{h}\right)$ level. Thus, planar corannulene (1) is described as a $4 \mathrm{~d}+4 \mathrm{p}$ system in the notation of [3]. In $\mathrm{D}_{6 \mathrm{~h}}$ coronene (2) the electrons of the $2 \mathrm{e}_{2 u}$ HOMO (RHF/ 6-31G** $-0.33 \mathrm{E}_{\mathrm{h}}$ ) have both a translational transition to the $3 \mathrm{e}_{\mathrm{lg}} \mathrm{LUMO}\left(\mathrm{RHF} / 6-31 \mathrm{G}^{* *} 0.07\right.$ $\left.\mathrm{E}_{\mathrm{h}}\right)$ and a rotational transition to the nearby $3 \mathrm{e}_{2 \mathrm{u}}$ $\mathrm{LUMO}+1$ (RHF/6-31G** $\left.0.13 \mathrm{E}_{\mathrm{h}}\right)$. The four HOMO $\pi$ electrons give rise to the complete 'rim and hub' current pattern and hence $\mathbf{2}$ is described as a $4 \mathrm{dp}$ system [3].

Fig. 3 shows the orbital contributions to the $\pi$ current density map for the planar structure of [7]circulene (3a). The 2e 2 HOMO (RHF/6-31G** $-0.25 \mathrm{E}_{\mathrm{h}}$ ) contributes the whole of the central paratropic current (Fig. 3a); the $2 \mathrm{e}_{3}^{\prime \prime} \mathrm{HOMO}-1$ (RHF/6-31G** $-0.30 \mathrm{E}_{\mathrm{h}}$ ) contributes the whole of the perimeter diatropic current (Fig. 3b). As Fig. $3 \mathrm{c}$ shows, the combined $2 \mathrm{e}_{2}^{\prime \prime}+2 \mathrm{e}_{3}^{\prime \prime}$ current density is indistinguishable from the full $\pi$ map. The remaining $20 \pi$ electrons make a negligible contribution to the $\pi$ current density in the plotting plane (Fig. 3d). The sense of circulation in the orbital maps is entirely explained by the frontier electronic structure of 3a. The HOMO-LUMO $\left(2 \mathrm{e}_{2}^{\prime \prime} \rightarrow 3 \mathrm{e}_{2}^{\prime \prime}\right)$ is rotationally but not translationally allowed and hence gives rise to a paratropic current, and conversely the HOMO - $1 \rightarrow$ LUMO + 1 excitation $\left(2 \mathrm{e}_{3}^{\prime \prime} \rightarrow 3 \mathrm{e}_{1}^{\prime \prime}\right)$ is a purely translational transition, generating a diatropic current [RHF/6$31 \mathrm{G}^{* *}$ LUMO $0.08 \mathrm{E}_{\mathrm{h}}\left(3 \mathrm{e}_{2}^{\prime \prime}\right)$ and LUMO +10.10 $\mathrm{E}_{\mathrm{h}}\left(3 \mathrm{e}_{1}^{\prime \prime}\right)$. There is also a weak rotationally and 

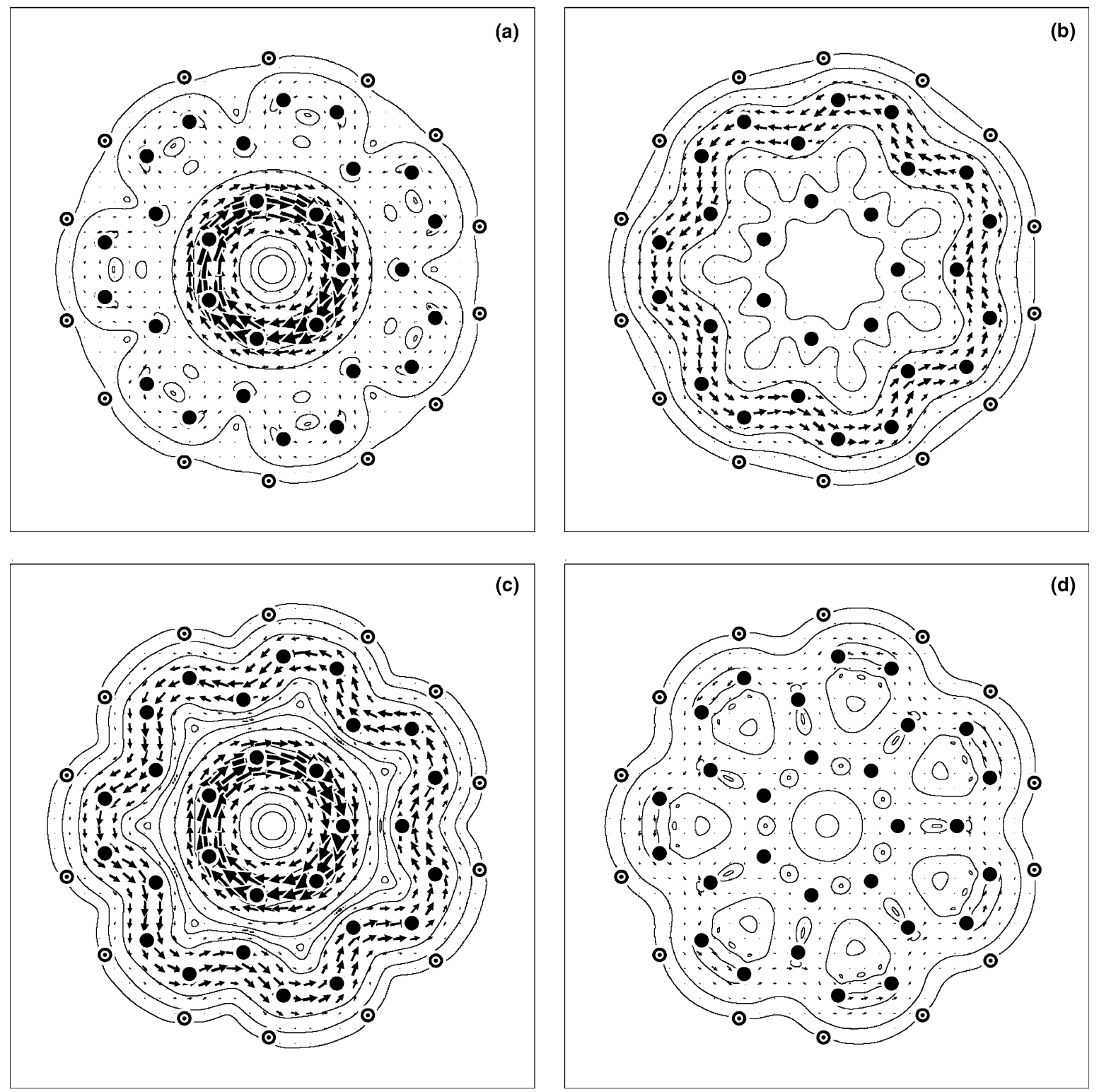

Fig. 3. Contributions to the $\pi$ current density maps for planar [7]circulene 3a $\left(\mathrm{D}_{7 \mathrm{~h}}\right)$; (a) doubly degenerate HOMO (2e $\left.\mathrm{e}_{2}^{\prime \prime}\right)$, (b) doubly degenerate HOMO - $1\left(2 \mathrm{e}_{3}^{\prime \prime}\right)$, (c) HOMO + HOMO - $1\left(2 \mathrm{e}_{2}^{\prime \prime}+2 \mathrm{e}_{3}^{\prime \prime}\right)$, and (d) all $\pi$ orbitals minus HOMO and HOMO -1 . In each case the plotting plane lies at a distance of $1 a_{0}$ from the median plane (carbon $\bullet$ and hydrogen $\odot$ ).

translationally allowed contribution from $\mathrm{HOMO} \rightarrow \mathrm{LUMO}+1 \quad\left(2 \mathrm{e}_{2}^{\prime \prime} \rightarrow 3 \mathrm{e}_{1}^{\prime \prime}\right)$. The spatial distribution of the induced currents follows from that of the molecular orbitals involved. Hence, planar [7]circulene 3a like the planar [5]circulene, corannulene (1), is a $4 d+4 p$ system.
As will be shown now, these considerations carry over without essential change to the saddleshaped [7] circulene $\mathbf{3 b}\left(\mathrm{C}_{2}\right.$ symmetry), in spite of the loss of formal $\sigma-\pi$ separability. Fig. 4 shows the combined orbital contributions of the four HOMO electrons of $\mathbf{3 b}$. Although an alternation 


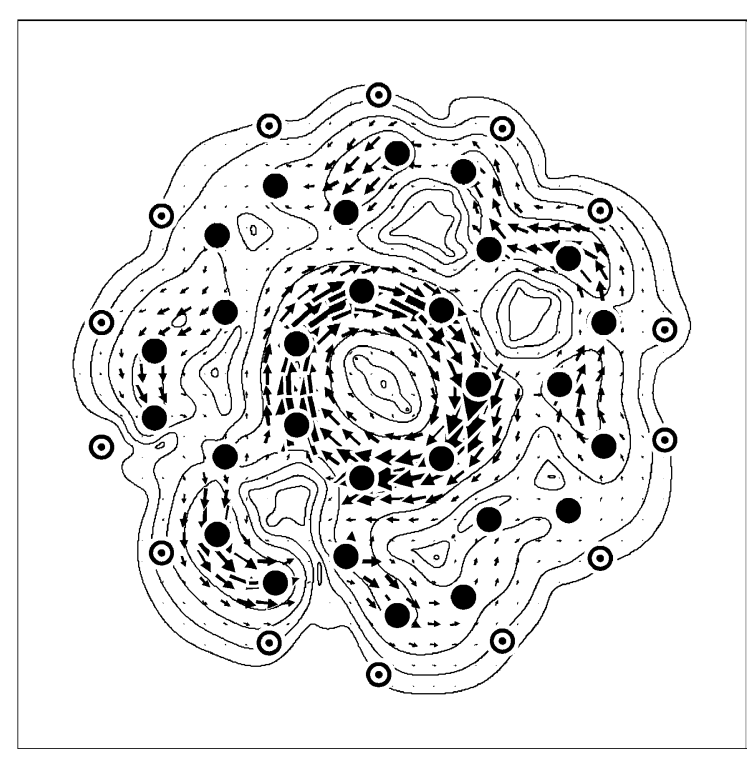

Fig. 4. Contribution of the HOMO (44b) and HOMO - 1 (47a) orbitals to the induced current density for saddle-shaped [7]circulene $3 \mathbf{b}\left(\mathrm{C}_{2}\right)$ plotted in a plane $1 a_{0}$ above the mean inertial plane (see text; carbon $\bullet$ and hydrogen $\odot$ ).

of intensity introduced by the mismatch of the planar plotting surface and the highly non-planar carbon framework is found, the central paramagnetic and the outer diamagnetic circulation are still clearly visible (see also Figs. 2 and 3). The topology of counter-rotating currents is not changed by the purely geometrical out-of-plane distortion of the molecule.

\subsection{Global and local magnetic properties of $3 \boldsymbol{a}$ and $3 b$}

Global molecular properties are consistent with the qualitative features displayed by the current density maps. For planar 3a and saddle-shaped $\mathbf{3 b}$ magnetisabilities $\xi$ of -43.1 and -48.1 a.u. were computed. These values are in the same range as those computed for the planar geometry of corannulene $(1, \xi-34.6$ a.u.) and for coronene $(2, \xi$ -51.1 a.u.). The sign of the anisotropy term $(\Delta \xi$ -71.7 a.u) for $\mathbf{3 a}$ is consistent with the diatropicity of the perimeter circulation and the classical $r^{2}$ scaling of $\xi$ for a current loop [2]. The variation of $\Delta \xi$ with $n$ is consistent with the relative strengths of the opposing central current $(\mathbf{1}, \Delta \xi-61.8$ a.u and $\mathbf{2}, \Delta \xi-101.6$ a.u [6]).

In Table 2 the computed CTOCD-PZ2 [19,20] ${ }^{13} \mathrm{C}\left(\delta_{\mathrm{C}}\right)$ and ${ }^{1} \mathrm{H}\left(\delta_{\mathrm{H}}\right)$ NMR chemical shifts for planar 3a and saddle-shaped $\mathbf{3 b}$ are presented and compared with available experimental values $[8,13]$. In agreement with the shallow corrugation potential for 3b ([14] and see Section 2) and the observation that $\mathbf{3 b}$ in solution possesses a timeaveraged planar $\mathrm{D}_{7 \mathrm{~h}}$ structure, even at $-90{ }^{\circ} \mathrm{C}$, a better fit between theory and experiment is obtained using the symmetry-averaged $\delta_{\mathrm{C}}$ values rather than those computed for the $\mathrm{D}_{7 \mathrm{~h}}$ struc-

Table 2

Chemical shifts $\left(\delta_{\mathrm{C}}\right.$ and $\left.\delta_{\mathrm{H}}\right)$ for $\mathbf{3 a}, \mathbf{3 b}$ and symmetry-averaged 3b [3b(av)]

\begin{tabular}{|c|c|c|c|c|}
\hline \multirow[t]{2}{*}{ Position $^{\mathrm{a}}$} & \multicolumn{4}{|l|}{$\delta_{\mathrm{C}}$} \\
\hline & $3 a$ & $3 \mathbf{b}$ & $3 b(a v)$ & $\delta_{\mathrm{C}, \exp }^{\mathrm{b}}$ \\
\hline 12 & & 140.1 & & \\
\hline 2 & & 135.7 & & \\
\hline 3 & 140.4 & 139.3 & 137.8 & 136.0 \\
\hline 4 & & 137.3 & & \\
\hline 5 & & 129.7 & & \\
\hline 6 & & 134.1 & & \\
\hline 7 & 136.0 & 130.6 & 132.3 & 132.1 \\
\hline 8 & & 133.5 & & \\
\hline 9 & & 127.9 & & \\
\hline 10 & & 126.4 & & \\
\hline 11 & & 126.3 & & \\
\hline 12 & 131.6 & 127.5 & 127.1 & 127.5 \\
\hline 13 & & 127.6 & & \\
\hline 14 & & 126.8 & & \\
\hline 15 & & 126.8 & & \\
\hline \multirow[t]{2}{*}{ Position $^{\mathrm{a}}$} & \multicolumn{4}{|l|}{$\delta_{\mathrm{H}}$} \\
\hline & $3 a$ & $3 \mathbf{b}$ & $3 b(a v)$ & $\delta_{\mathrm{H}, \exp }^{\mathrm{b}}$ \\
\hline 9 & & 7.4 & & \\
\hline 10 & & 6.9 & & \\
\hline 11 & & 7.0 & & \\
\hline 12 & 7.2 & 7.4 & 7.1 & 7.5 \\
\hline 13 & & 7.2 & & \\
\hline 14 & & 7.0 & & \\
\hline 15 & & 7.2 & & \\
\hline
\end{tabular}

The calculated mean nuclear shieldings $\sigma_{\mathrm{C}}$ and $\sigma_{\mathrm{H}}$ are converted to predicted chemical shifts by the relations [25], $\delta_{\mathrm{C}}=185.6-\sigma_{\mathrm{C}}(\mathrm{av}) \times 10^{6}$ and $\delta_{\mathrm{H}}=30.8-\sigma_{\mathrm{H}}(\mathrm{av}) \times 10^{6}$ (in $\mathrm{ppm})$.

${ }^{\mathrm{a}}$ See Fig. 1.

${ }^{\mathrm{b}}$ See $[8,13]$. 
ture itself, which show a systematic devation of 4 ppm from experiment (see Table 2). The computed $\delta_{\mathrm{H}}$ value for $3 \mathbf{a}(7.2 \mathrm{ppm})$ and the corresponding symmetry-averaged value for $\mathbf{3 b}$ (7.1 ppm) are nearly identical (Table 2), and both are in satisfactory agreement with experiment [ $\delta_{\mathrm{H}} 7.5 \mathrm{ppm}$ (solvent $\mathrm{CD}_{2} \mathrm{Cl}_{2}$ ) [8,13]] (cf. also [6]).

We note in passing that the absolute shieldings computed at the ring centres (apart from sign, equal to NICS [25]) are compatible with the rim and hub current pattern, i.e. the hexagons $\boldsymbol{A}-\boldsymbol{D}$ have values similar to benzene itself $[\mathbf{3 a}, \sigma(\boldsymbol{A}-$ $\boldsymbol{D})=11.0 \mathrm{ppm}, \quad \mathbf{3 b}, \quad \sigma(\boldsymbol{A})=10.7, \quad \sigma(\boldsymbol{B})=10.2$, $\sigma(\boldsymbol{C})=11.0$ and $\sigma(\boldsymbol{D})=10.1 \mathrm{ppm}$, and $\mathbf{3 b}(\mathbf{a v})$, $\sigma(\boldsymbol{A}-\boldsymbol{D})=10.5 \mathrm{ppm}]$ and the central heptagonal ring has a shielding of the opposite sign [3a, $\sigma(\boldsymbol{E})=-11.6 \mathrm{ppm}$ and $\mathbf{3 b}, \sigma(\boldsymbol{E})=-10.8 \mathrm{ppm}]$, in agreement with the strong central paratropic current (Figs. 2 and 3). As the isotropic average shieldings in $\mathbf{3 a}$ and $\mathbf{3 b}$ show, this pattern is not significantly disturbed by the loss of planarity in $3 b$.

\subsection{A simple graph-theoretical model}

Given that the ab initio computed maps show such strong similarities in ring-current pattern between $[n]$ circulenes with $n=5(1), 6$ (2) and 7 (3), independently of the planarity or non-planarity of the molecular structure, it is natural to ask whether the opposed rim and hub pattern is a consequence of the $[n]$ circulene topology itself. If so, a purely graph-theoretical model should be able to rationalise this behaviour. The simplest explicit model of this kind for magnetic response is the venerable Hückel-London model [22,24].

Fig. 1 shows a schematic diagram of the molecular graph of the $[n]$ circulene carbon skeleton. In maximal $\mathrm{D}_{n \mathrm{~h}}$ symmetry, the graph has four symmetry-distinct sets of edges and hence in principle four independent $\beta$ bond parameters, $\beta_{1}-\beta_{4}$, in a Hückel treatment. The crude Hückel model takes all $\beta$ parameters to be equal and yields orbital energies $\epsilon_{i}=\alpha+\lambda_{i} \beta$, where the $\lambda_{i}$ are the $4 n$ eigenvalues of the adjacency matrix of the unweighted $[n]$ circulene graph. This model predicts a closed-shell $\pi$ configuration for all $[n]$. In a more detailed treatment, differences between the distinct $\beta$ values can be introduced, and in particular if 'spokes' and 'other' edges are distinguished $\left(\beta_{2}=\beta_{\text {spoke }}, \beta_{1}=\beta_{3}=\beta_{4}=\beta_{\text {other }}\right)$ the Hückel model can represent a continuum of descriptions from the $[n]$ annulene-within-an- $[m]$ annulene $\left(\beta_{\text {spoke }} / \beta_{\text {other }}=0\right)$ to the fully coupled circulene $\left(\beta_{\text {spoke }} / \beta_{\text {other }}=1\right)$. The ratio $\left(\mu=\beta_{\text {spoke }} /\right.$ $\beta_{\text {other }}$ ) plays the role of a coupling parameter, tracking the relative strength of radial and tangential bonds.

The patterns of currents induced in the structure by a perpendicular magnetic field directed along the $\mathrm{C}_{n}$ axis can be calculated as a function of the coupling strength by invoking the HückelLondon model $[22,24]$. Computation of the vector potential in this model requires physical coordinates for bond centres, which can be estimated by assuming equilateral bonds all with the idealised benzene length of $1.40 \AA$. The results of the Hückel-London calculation follow the same general pattern for all $\mathrm{n}$ as illustrated for $n=5,6$ and 7 in Fig. 5.

At zero coupling strength $\left(\beta_{\text {spoke }}=0\right)$ the system consists of two uncoupled annulenes: an $[n]$ annulene-within-an- $[m]$ annulene, which will be $4 n+1$ in $4 m^{\prime}+3,4 m+2$ in $4 m^{\prime}+2,4 m+3$ in $4 m^{\prime}+1$ or $4 m$ in $4 m^{\prime}$ according to the divisibility of $n$. From the analytical Hückel solutions for the annulenes, the first three cases give closed shell systems, and the fourth gives a closed shell for any small but non-zero $\beta_{\text {spoke. The Hückel-London }}$ currents are therefore con-rotating and diatropic on both rings. As the coupling parameter $\mu$ increases at constant $\beta_{\text {other }}$, the perimeter remains diatropic, but the hub current first weakens, vanishes at some critical $\mu$, and then grows back with a reversed sense. The critical value changes with $n$ but ultimately as $\mu$ approaches 1 all $[n]$ circulenes conform to the counter-circulating, rim and hub pattern. Thus, in the strongly coupled regime characteristic of the physical systems, predictions of the $[n]$ annulene-within-an- $[m]$ annulene model are overturned. A 'mechanical' model based on these principles has recently been shown to rationalize patterns of local aromaticity in (isolated pentagon) fullerene molecules where pentagonal 

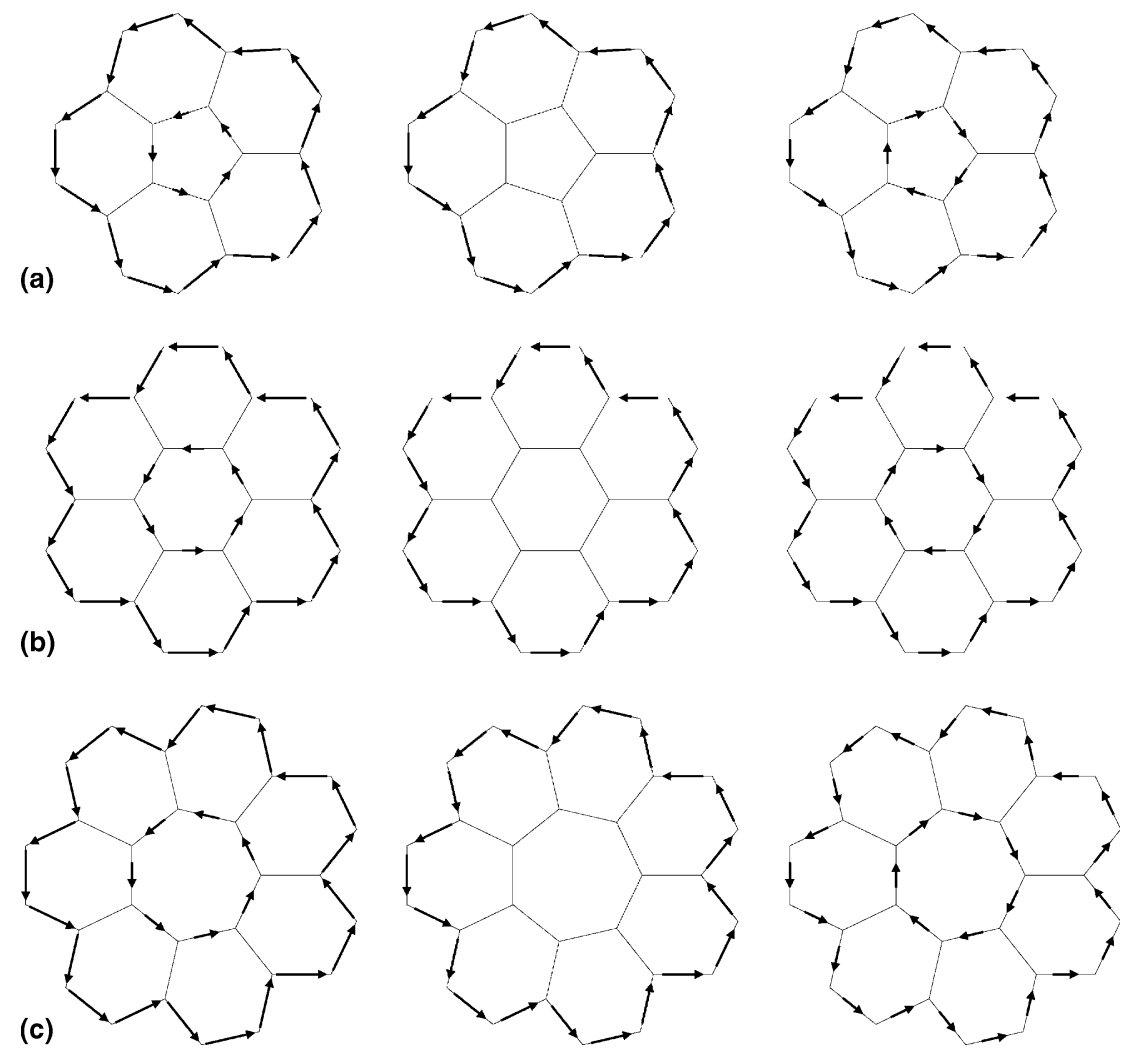

Fig. 5. Hückel-London predictions of ring-current patterns in $[n]$ circulenes. The cases of weak, intermediate and strong coupling (left, centre, right) are illustrated for (a) $n=5$ (1), (b) $n=6$ (2), and (c) $n=7$ (3).

rings are similarly circumscribed by circuits of hexagons [26].

\section{Conclusions}

The ab initio current density maps for planar [3a $\left(D_{7 h}\right)$ ] and saddle-shaped [7]circulene [3b $\left(C_{2}\right)$ ] show that 3a-b sustain a similar global pattern of counter-rotating rim (diatropic) and hub (strongly paratropic) currents as found previously for both planar $\left(\mathrm{D}_{5 \mathrm{~h}}\right)$ and bowl-shaped $\left(\mathrm{C}_{5 \mathrm{v}}\right)$ corannulene (1) [6]. The breakdown of the computed current density of $\mathbf{3 a}-\mathbf{b}$ into orbital contributions reveals that 3, like 1 [3], is a $4 d+4 p$ system with eight magnetically active and 20 magnetically inactive $\pi$ electrons. Thus, for $\mathbf{3}$, as for $\mathbf{1}$ and $\mathbf{2}$, the proposition that $[n]$ circulenes behave magnetically as decoupled annulenes is not tenable. A comparison of the calculated integrated magnetic properties $\left(\delta_{\mathrm{C}}\right.$ and $\delta_{\mathrm{H}}, \Delta \xi$, and NICS values) for $\mathbf{3 a}$ and $\mathbf{3 b}$ indicates that the best fit to available experimental data $[8,13]$ is obtained by symmetry averaging of the properties of the individual sites of the non-planar form $\mathbf{3 b}$, which is consistent with a picture of $\mathbf{3}$ as a rapidly inverting extremely floppy species which spends much of its life in out-of-plane geometries.

The similarity of the ab initio current density maps of $[n]$ circulenes for $n=5$ (1), 6 (2) [6], 7, and 18 (kekulene, [16]), is rationalised by a simple, purely graph-theoretical model, based on HückelLondon theory. The general pattern of circulation in an $[n]$ circulene is described by a one-parameter model that gives a continuum description from the fully decoupled con-rotating currents of the $[n]$ annulene-within-an- $[m]$ annulene to the more physically realistic fully coupled counter-rotating currents of the real $[n]$ circulene. 


\section{Acknowledgements}

We acknowledge financial support from the European Union TMR Marie-Curie Training Site schemes [contracts FMRX-CT097-0192 (R.W.A.H.) and HPMT-CT-2000- 00016 (A.A.)].

\section{References}

[1] J.A. Pople, J. Chem. Phys. 24 (1956) 1111

[2] H.J. Dauben, J.D. Wilson, J.L. Laity, Diamagnetic Susceptibility Exaltation as a Criterion of Aromaticity, in: J.P. Snijder (Ed.), Nonbenzenoid Aromatics, vol. II, Academic Press, New York, 1971, p. 167.

[3] E. Steiner, P.W. Fowler, J. Phys. Chem. A 105 (2001) 9553.

[4] T.A. Keith, R.F.W. Bader, Chem. Phys. Lett. 210 (1993) 223.

[5] S. Coriani, P. Lazzeretti, M. Malagoli, R. Zanasi, Theoret. Chim. Acta (89) (1994) 181

[6] E. Steiner, P.W. Fowler, L.W. Jenneskens, Angew. Chem. Int. Ed. Engl. 40 (2001) 362.

[7] Cf. J.S. Siegel, T.J. Seiders, Chem. Britain (1995) 313.

[8] K. Yamamoto, T. Harada, Y. Okamoto, H. Chikamatsu, M. Nakazaki, Y. Kai, T. Nakao, M. Tanaka, S. Harada, N. Kasai, J. Am. Chem. Soc. 110 (1988) 3578.

[9] J.K. Fawcett, J. Trotter, Proc. R. Soc. A 289 (1965) 366.

[10] J.C. Hanson, C.E. Nordman, Acta Cryst. B 32 (1976) 1147.

[11] L.T. Scott, M.M. Hashemi, M.S. Bratcher, J. Am. Chem. Soc. 114 (1992) 1920.
[12] A. Borchardt, A. Fuchicello, K.V. Kilway, K.K. Baldridge, J.S. Siegel, J. Am. Chem. Soc. 114 (1992) 1921.

[13] M. Sato, K. Yamamoto, H. Sonobe, K. Yano, H. Matsubara, H. Fujita, T. Sugimoto, K. Yamamoto, J. Chem. Soc., Perkin Trans. 2 (1998) 1909.

[14] M. Shen, I.S. Ignatyev, Y. Xie, H.F. Schaefer III, J. Phys. Chem. 97 (1993) 3212.

[15] See also J.H. Dopper, H. Wynberg, J. Org. Chem. 40 (1995) 1957.

[16] E. Steiner, P.W. Fowler, L.W. Jenneskens, A. Acocella, Chem. Commun. (2001) 659.

[17] M.F. Guest et al., It is derived from the original GAMESS code due to M. Dupuis, D. Spangler, J. Wendolowski, NRCC Software Catalog, Vol. 1, Program No. QG01 (GAMESS) 1980.

[18] P. Lazzeretti, R. Zanasi, SYSMO package (University of Modena), 1980, with additional routines for evaluation and plotting of current density by E. Steiner, P.W. Fowler (University of Exeter, UK), unpublished results.

[19] R. Zanasi, P. Lazzeretti, M. Malagoli, F. Piccinini, J. Chem. Phys. 102 (1995) 7150.

[20] R. Zanasi, J. Chem. Phys. 105 (1996) 1460.

[21] A. Soncini, P.W. Fowler, I. Černuv̌sak, E. Steiner, Phys. Chem. Chem. Phys. 3 (2001) 3920.

[22] A. Pasquarello, M. Schlüter, R.C. Haddon, Phys. Rev. A 47 (1993) 1783.

[23] P. Lazzeretti, Prog. Nucl. Magn. Res. 36 (2000) 1.

[24] F. London, J. Phys. Radium 8 (1937) 397.

[25] P. von, R. Schleyer, C. Maerker, A. Dransfeld, H. Jiao, N.J.R. van Eikema Hommes, J. Am. Chem. Soc. 118 (1996) 6137-6318.

[26] G. van Lier, P.W. Fowler, F. De Proft, P. Geerlings, J. Phys. Chem. A. 106 (2002) 5128. 